Research Article

\title{
Industry Development of Derivative Functionalized Gold Nanomaterials and Their Application in Chemiluminescence Bioanalysis: Based on the Industrial Practice of China's Central Yunnan Urban Agglomeration
}

\author{
Tengfei Ma ${ }^{1}$ and $\mathrm{Hao} \mathrm{Wu}^{2}{ }^{2}$ \\ ${ }^{1}$ Faculty of Management and Economics, Kunming University of Science and Technology, Kunming 650093, Yunnan, China \\ ${ }^{2}$ Faculty of Materials Science and Engineering, Kunming University of Science and Technology, Kunming 650093, Yunnan, China \\ Correspondence should be addressed to Hao Wu; luck@stu.kust.edu.cn
}

Received 22 July 2020; Revised 27 August 2020; Accepted 28 September 2020; Published 23 October 2020

Academic Editor: Tifeng Jiao

Copyright (C) 2020 Tengfei Ma and Hao Wu. This is an open access article distributed under the Creative Commons Attribution License, which permits unrestricted use, distribution, and reproduction in any medium, provided the original work is properly cited.

\begin{abstract}
Electrochemiluminescence biosensor is an analytical method combining electrochemiluminescence technology with biosensor. Using nanomaterials as electrochemical luminescence sensor platform can not only immobilize a large number of biomolecules but also improve the performance of the sensor to realize the supersensitive detection of biomacromolecules. Although these methods have high sensitivity for bioanalysis, there are still some shortcomings which limit the practical application. Therefore, this paper discusses the development of functional gold nanomaterials industry and its application in chemiluminescence bioanalysis. In this paper, two methods of synthesizing luminescent functional gold nanomaterials at room temperature were studied by using chemiluminescent reagents as reducing agent and protective agent. Based on luminescent functionalized gold nanoparticles, immunoassay and DNA bioanalysis probes were constructed, and their applications in chemiluminescence and electrochemiluminescence bioanalysis were discussed. Finally, the simulation results show that the relative deviation between the experimental results and the existing clinical methods is less than $17 \%$. The sensor has good stability and selectivity and can be used for the determination of CEA in human serum. The gold nanomaterials synthesized by further research have excellent chemiluminescence activity and can be used to label biomolecules and prepare biological probes. This article aims to explore the application of chemical methods in the transformation of new industries, to achieve breakthroughs in new products in industrial innovation, and to achieve the cross-fusion of management science and engineering disciplines and chemical disciplines. The industrial development of derivative functionalized gold nanomaterials has broad application prospects in biological analysis.
\end{abstract}

\section{Introduction}

Electrochemiluminescence (ECL) is a kind of chemiluminescence phenomenon caused by the electrochemical method. Some special substances are produced on the electrode surface by applying a certain voltage or a certain current. These special substances or other coexisting components in the system form high-energy excited states through electron transfer, and the excited states transits back to the ground state. As a new material, gold nanomaterials can serve the development of emerging industries. When developing emerging industries, Yunnan Province, China, focuses on eight key industries and actively builds three world-class brands. In this process, the development of gold nanomaterials industry is very important. Good bonding point: in recent years, the conversion of new and old kinetic energy in Yunnan Province has accelerated. Eight key industries such as biomedicine and general health, as well as the development of green energy, green food, and healthy living destinations "three cards" have become a new driving force for Yunnan's economic development. In the work of the modern industrial system oriented to 2035 , it is crucial to 
understand the specific properties of gold nanomaterials and their application in chemiluminescence bioanalysis.

Electroluminescence (ECL) is generally based on a three electrode system, which is connected by wires, respectively. The ECL signal changes on the working electrode surface are detected by photomultiplier tube or charge coupled element, so as to realize quantitative or qualitative analysis $[1,2]$. Amine-rich nitrogen-doped carbon nanoparticles have been successfully used as coreactants in the electrochemiluminescence process. Carrara identified the primary or tertiary amino groups on NCNDs as coreaction sites for $\mathrm{Ru}$ (bpy) $32+\mathrm{ECL}$, indicating that they are powerful substitutes for tripropylamine. Carrara also reported the synthesis and electrochemiluminescence behavior of NCNDs covalently bonded with Ru (bpy) $32+$. NCNDs in the hybrid are not only ECL-labeled carriers but also coreactants for ECL production. The results show that the ECL emission of the hybrid product is higher than that of the single component combination, indicating that the self enhanced ECL of ruthenium complex is due to the intramolecular electron transfer process [3, 4]. Liu constructed a label-free electrochemiluminescence sensor for the detection of acetylcholinesterase using gold nanoparticles functionalized g-c3n4 nanocomposite as light-emitting carrier. Liu et al. used au-g-c3n4-nh and thiol-modified ache-specific aptamer-modified glassy carbon electrode to prepare the sensor. In the presence of acetylcholinesterase, acetylcholinesterase can hydrolyze the substrate acetylcholine to produce acetic acid, which reacts with triethylamine, leading to the consumption of CO reactant. The ECL response of the sensor was linear with the concentration of ache in the range of $0.1 \mathrm{pg} / \mathrm{ml} \sim 10 \mathrm{ng} / \mathrm{ml}$, and the detection limit was $42.3 \mathrm{FG} /$ $\mathrm{ml}$. This novel ECL sensing strategy provides a highly sensitive and selective method for ache detection and is expected to have potential application prospects in clinical diagnosis and biomedical technology $[5,6]$. Zhang proposed a small molecule dye Alexa flow 488 as the donor and electrochemiluminescence resonance energy transfer strategy. Zinc Cadmium selenium quantum dots were used as acceptors to construct a DNA nanomachine-based regenerative biosensor for ultrahigh sensitivity cancer cell detection without any enzyme. Zhang et al.'s research shows that it can be used for on-site environmental analysis and monitoring, thus providing a new modular platform for the construction of functional DNA nanomachines, which can be used for the ultrahigh sensitivity analysis of promising biomarkers and toxic metals $[7,8]$. Zhang et al. studied the electrochemiluminescence (CL) behavior of mesoporous tetraporphyrin in strong and stable emission aqueous solution and determined the nanomolar concentration of copper ion. Zhang et al. proposed two possible reaction mechanisms to understand the mechanism of ECL formation in TSPP/K2S2O8 system. Zhang et al. proposed and successfully applied it to the electrochemiluminescence analysis of tap water samples. Zhang et al. predicted that TSPP would be a new type of ECL luminescent material $[9,10]$. A new type of double stabilizer-capped CdSe quantum dots with unique electrochemiluminescence properties was prepared by Jie, and it was used for the first time to detect thrombin with high sensitivity by the targettriggered multiple amplification method. Ji et al.'s research shows that this flexible biosensor system has good performance in the detection of human serum and has a wider application prospect than other systems based on ECL reagent $[10,11]$.

With the rapid development of nanomaterials, self-luminescent nanomaterials such as quantum dots and metal nanoclusters have attracted extensive attention and have been widely used in the construction of biosensors $[12,13]$. The sensitivity, selectivity, and stability of ECL biosensor can be effectively improved by using the special properties of functionalized nanomaterials $[14,15]$. Silica nanoparticles with unique morphology can be used as an effective template to prepare silicon metal hybrid nanomaterials, which has a wide range of applications in many fields. Mesoporous silica nanoparticles with high-specific surface area and abundant pores can be used to synthesize mesoporous silica core metal shell nanostructures with catalytic activity centers. Wongyun et al. have successfully synthesized DFNS/AU hybrid nanomaterials using high-specific surface area dendritic fiber nanosilicon as template. Gold nanoparticles were initially synthesized on the surface of DFNS by selective reduction of gold ions after surface modification. Wongyun et al. used the seed-mediated growth method to grow $\mathrm{Au}$ nanoparticles on the DFNS/Au sites to generate DFNS core $\mathrm{Au}$ nanoparticles shell hybrid and DFNS core Au layer shell hybrid. Wongyun et al. compared the catalytic activity of DFNS/Au nanoparticles and DFNS/Au layers in the reduction of 4-nitrophenol $[16,17]$. Ushakov studied the effect of pore structure on the height of electron barrier in $\mathrm{Au}$ $\mathrm{Al}_{2} \mathrm{O}_{3}$ structure. Ushakov studied the pore structure of two types of anodic alumina: through-pass and pass. The results show that the deposited Au films produce electron barriers with different heights at the metal insulator interface, which depends on the shape of the hole profile. Linear optical spectroscopy showed that the barrier height with throughhole structure was increased by $2.0 \mathrm{EV}[18,19]$. It is difficult to preserve the properties of gold nanoparticles in the form of solution and dry powder for a long time. Fernández Lodeiro et al. synthesized gold nanoparticles using organic tellurium derivatives as reducing agents and stabilizers. Fernández Lodeiro et al.'s research shows that the organic tellurium shell can be easily removed by the resuspension of nanoparticles in environmentally friendly solvents, so that the gold core can be used for subsequent applications $[20,21]$. Liu prepared gold nanomaterials with different morphologies by the wet chemical reduction method and characterized by transmission electron microscope and ultraviolet visible spectrophotometer. TEM results show that $\mathrm{Au}$ nanomaterials with different morphologies, such as spherical, rod-shaped, cubic, and triangular, have been prepared. The UV absorption spectra of au-24-23 are given. Some nanomaterials can be used as coreaction reagents to effectively enhance the luminescence of the ECL system [22].

Guang discussed the development trend of intelligent robots and put forward some thoughts and suggestions on the development of intelligent robots in China. The main technologies of intelligent robots at home and abroad are 
summarized, and the relevant theories, methods, and technical guidance of intelligent robot technology and industrial development are provided. The design and synthesis of photocatalysts for efficient degradation of organic pollutants in China are considered to be a promising way for environmental protection and governance. The photodegradation efficiency of the optimized BiVO4/NIS/Au ternary composite for tetracycline is 4.25 times higher than that of pure BiVO4. Through capture experiments and liquid chromatography-mass spectrometry (LC-MS) analysis, Zhang et al. proposed a possible degradation mechanism and pathway $[23,24]$. Simin et al. proposed a layer by the layer self-assembly method. The nanostructures composed of hydrophobic CdSe/CdZnS and hydrophilic CdTe and $\mathrm{Au}$ nanoparticles were formed on the slide by a layer of functional silica. In contrast, when the glass layer thickens, a bright PL appears. LBL self-assembled glass film has good stability and transparency and has broad application prospects in biochemical, photoelectric, clinical diagnosis, and other fields [25]. Ruirui can spontaneously form injectable and self-healing collagen-gold hybrid hydrogels via electrostatic self assembly and subsequent biomineralization. Cuihong proposed a two-step method for the synthesis of perovskite quantum dots and large-size nanoparticles (LNPS). The growth of QDs and LNPS involves two possible pathways, namely, the rapid intercalation reaction process and the dissolution recrystallization process. On the basis of a comprehensive understanding of its formation mechanism, Cuihong partially realized the transformation from LNPS to quantum dots by properly adding surfactants, which is expected to pave the way for the development of pure production of perovskite quantum dots [26].

In order to improve the sensitivity and biocompatibility of ECL biosensor, a new type of low toxicity self-luminescent nanomaterial was introduced as ECL reagent and functional nanomaterial was used as carrier of biomolecule and luminescent reagent. Combined with the immunosandwich analysis method and target induced shear, ECL biosensor with good stability, high sensitivity, and low toxicity was constructed. The construction of sensors, enhancement of electroluminescence signal, exploration of electroluminescence mechanism, and detection performance of biosensors are discussed. Compared with other chemiluminescence immunoassay methods with gold nanoparticles, the labeling process is simple, fast, and cheap. In principle, the sensor can be used to detect other biomolecules based on antigen and antibody.

\section{Derivative Functionalized Gold Nanomaterials and Chemiluminescence Bioanalysis}

2.1. Derivative Functionalized Gold Nanomaterials. Nanomaterials are new materials with at least one-dimensional size between $1-100 \mathrm{~nm}$ in three-dimensional space. Because of its various characteristics, it has unique electrical and optical properties and catalytic activity. Nanomaterials with large specific surface area can support a large number of antibodies or luminescent reagents, the conductive nanomaterials can promote the electron transfer in the reaction process, and the good catalytic nanomaterials can promote the ECL reaction and speed up the reaction rate. These nanomaterials can enhance the stability of the sensor or improve its sensitivity. Nanomaterials have the characteristics of large specific surface area, good biocompatibility, and easy to modify and assemble. Therefore, nanomaterials can be used as carriers to improve the immobilization efficiency of luminescent reagents or biomolecules, so as to improve the sensitivity of sensors.

Conductive polymer/metal nanocomposites, combined with the dual advantages of organic materials and inorganic materials, have composite properties that other materials do not have. Using macromolecular acids and polymers as dopants, aniline was polymerized with p-aminothiophenol functionalized gold colloid in the aqueous system. Due to the high conductivity of $\mathrm{Au}$ and the strong interfacial effect between the two phases, the electrochemical properties of the materials are obviously enhanced. The composite materials with various morphologies can be obtained by in situ polymerization. The conductive polymer monomer and metal oxide were added into the dispersion, then the oxidant was added to initiate polymerization, and the composite was obtained after purification. In the process of synthesis, the change of each parameter may change the final morphology of nanocomposites. Transition metal oxides are one of the inorganic nanomaterials. Transition metals represent a series of metal elements in the $\mathrm{D}$ region of the periodic table, which have an unfilled valence layer of $\mathrm{d}$ orbitals and are easy to form metal complexes. In addition to gold and silver, most transition metals exist in the earth's crust in the form of sulfides or oxides. Transition metal oxide is the compound obtained by the combination of oxygen atom and transition metal. Its nanostructure has the characteristics of common nanomaterials. Due to its good biocompatibility, polyvinyl alcohol is considered as a kind of bionic material with excellent performance and is widely used in medical treatment. In theory, the preparation of polymer is made by monomer polymerization, but "aterials. Due is easy to produce isomerization to acetaldehyde, so it is not easy to prepare polyvinyl alcohol according to the theory. They can only be prepared by alcoholysis or hydrolysis of polyvinyl acetate. The PVA prepared by alcoholysis method not only has high purity but also has good performance.

In a broad sense, biomaterials refer to the applied materials or materials that can contact with organisms through a series of biological processes; in a narrow sense, biomaterials refer to a kind of natural or synthetic special functional materials that can contact with the physiological environment of organisms, have strong biocompatibility, and can diagnose, replace, and repair their cells, tissues, and organs, or enhance their functions. So, biomaterials are also called biomedical materials. Bionic design is not only required to be similar to biological objects in structure but also to achieve certain similarity in function. The fracture property of shell is much stronger than that of ceramic. It is found that the main reason is the strength and toughness of nacre in the three- 
layer structure. The arrangement of aragonite crystal and organic matrix in nacre layer alternately makes it form multilayer toughened structure with alternating soft and hard layers. Organic matrix is easy to induce the deflection of vertical cracks in the outer layer, thus preventing the crack growth. This is the key to the high fracture toughness of nacre. The water droplets on the surface of lotus leaf will automatically gather and take away the dust on the surface. This superhydrophobic property has been widely used in self-cleaning, antipollution, and antiadhesion. Botanists have studied thousands of leaves. The superhydrophobicity is mainly determined by the micromorphology and surface energy of the surface. There are many papillary microconvex bodies on the surface of lotus leaf. The gap of micron scale is filled with air, and the outer area of milky convex surface is covered by many nanorods with low surface energy. This is the reason why water can not soak lotus leaves. Zheng Zhenrong et al. prepared PVDF films with nano-/micron bilayer structure by deposition method, which reached a contact angle of $157^{\circ}$ and increased the surface hydrophobicity.

2.2. Chemiluminescence. In other spectral quantitative methods, not only external light source is needed but also the factors that affect the signal-to-noise ratio, which are not necessary for chemiluminescence analysis. Since each chemiluminescence process includes the process of chemiluminescence reaction, the rate of chemical reaction, the yield of material that can be excited as luminescent body, and the luminous efficiency of the luminescent body itself have a crucial impact on the intensity of chemiluminescence. The chemiluminescence detection method is based on the luminous intensity of a certain time to detect the object. The $I_{\mathrm{CL}}$ at a given time $t$ can be expressed by the following equation:

$$
I_{\mathrm{CL}}(t)=\varphi_{\mathrm{CL}} \frac{\mathrm{d} c(t)}{\mathrm{d} t}=\varphi_{\mathrm{EX}} \varphi_{\mathrm{EM}} \frac{\mathrm{d} c(t)}{\mathrm{d} t},
$$

where $\mathrm{d} c(t) / \mathrm{d} t$ represents the chemiluminescence reaction rate at a specific time, $\varphi_{\mathrm{CL}}$ represents the quantum yield of chemiluminescence, and $\varphi_{\mathrm{EX}}$ and $\varphi_{\mathrm{EM}}$ represent the excited state quantum yield and luminescence quantum yield, respectively. $\varphi_{\mathrm{CL}}$ can be regarded as a constant in the chemiluminescence reaction, but factors such as $\mathrm{pH}$, ionic strength, temperature, and components contained in the solution have an important influence on the determination of chemiluminescence, which also leads to any quantum factor in the chemical reaction and can interfere with the intensity of chemiluminescence. Therefore, the concentration of a substance in a chemical reaction system can be determined by measuring the intensity of chemiluminescence under certain reaction conditions. That is, the quantitative analysis of chemiluminescence can be applied by the formula shown in (1) in a first-level or quasi-first-level chemical reaction. In the actual chemical reaction, when only the concentration of the substance $\mathrm{M}$ changes, the concentration of $\mathrm{M}$ can be measured by the signal value of chemiluminescence through the following formula:

$$
I_{\mathrm{CL}}=\int I_{\mathrm{CL}}(t) \mathrm{d} t=\int \varphi_{\mathrm{CL}}\left[\frac{\mathrm{d} C_{A}(t)}{\mathrm{d} t}\right] \mathrm{d} t=\varphi_{\mathrm{CL}} C_{A} .
$$

The chemiluminescence analysis method can be used to determine the substances that can enhance or inhibit the chemiluminescence reaction, the reactants in the chemiluminescence reaction system or the substances produced in the reaction, and some substances that can produce coupling effect in the chemiluminescence reaction.

Some compounds with oxidation or fluorescence properties and aromatic oxalates are the main components in the chemiluminescence reaction system of oxalate peroxide. The effect of compounds with fluorescence properties in this system is to improve the stability and weak luminescence of the luminescent system of oxalate peroxide and oxidant, and the fluorescence spectrum of the fluorescent material itself is consistent with that in the chemiluminescence system. The energy transfer luminescence system is the most efficient chemiluminescence system at present. Different from luminol and other chemiluminescent systems, oxalate peroxides are characterized by energy transfer. The main applications of oxalate chemiluminescence system include that the chemiluminescence emitted by the system is not only high intensity but also the emission wavelength can be controlled within a certain range, so the development of multimode light sources has been industrialized; oxalate ester luminescent system has been widely recognized in chemiluminescence analysis. For the determination of hydrogen peroxide, enzyme reactants, and fluorescent substances, it is a typical analytical application.

\subsection{Chemiluminescence Catalyst and Biosensor. Metal} nanoparticles are widely used in the field of analytical sensing due to their unique structure and excellent physical and chemical properties. Among them, gold, silver, and platinum nanoparticles are the most common catalysts in chemiluminescence analysis. Generally, gold is considered to be an inert metal material. However, the properties of gold nanoparticles are quite different and show good catalytic activity. Therefore, gold has become the first nanomaterial for chemiluminescence analysis. In addition to the luminol hydrogen peroxide chemiluminescence system, gold nanoparticles can also catalyze luminol the $\mathrm{NaIO}_{4}$ system and luminol $\mathrm{AgNO}_{3}$ system. Compared with gold nanoparticles, silver nanoparticles have stronger catalytic activity for the luminol hydrogen peroxide system. It can be used for the selective determination of cysteine because of its adsorption on Ag nanoparticles and competitive consumption of active intermediates. Platinum nanoparticles and alloys also have good catalytic activity for luminol hydrogen peroxide.

As a member of the family of carbon nanomaterials, carbon dots mainly refer to photoluminescence carbon nanoparticles less than $10 \mathrm{~nm}$, which have the characteristics of surface modification or functionalization and can be rapidly synthesized and functionalized. As a catalyst, carbon dots effectively catalyze the decomposition of dissolved oxygen to produce superoxide radicals, which accelerates the 
electron transfer rate between dissolved oxygen and luminol, resulting in enhanced chemiluminescence. The good catalytic activity of carbon point is closely related to its surface state and particle size. MOFs have a large number of unsaturated metal sites, showing good catalytic activity, and can be used for quantitative detection of a variety of analytical substances. MIL-53 (FE) has good chemiluminescence activity, which can catalyze luminol hydrogen peroxide system to produce enhanced chemiluminescence, which can be used for the determination of glucose in human serum. In addition, MOFs with large specific surface area can also be used as a support for stabilizing and dispersing the catalyst, so as to improve the catalytic activity. The dispersion of MOFs used in luminol chemiluminescence system is poor in water, and more MOFs with good chemiluminescence activity can be explored for use in chemiluminescence system. Therefore, it is of great significance to improve the dispersion and catalytic activity of MOFs in water by surface modification. Although most MOFs contain metal ions $(\mathrm{Fe}, \mathrm{Ni}$, and $\mathrm{CO}$ ) as catalytic sites, their catalytic activity can be further enhanced by carbonization. The carbon material prepared by MOFs as sacrificial template has a large specific surface area, which can expose more active sites. At the same time, the doping of heteroatoms into the carbon matrix can improve the catalytic activity. Therefore, it is of great significance to prepare catalysts with higher catalytic activity for the luminol chemiluminescence system based on the existing MOFs.

ECL immunosensor is a biosensor composed of antigen, antibody, and other immune recognition elements. It combines the high sensitivity of the ECL analysis method and the high specificity of immune recognition. ECL reagent can be immobilized on the electrode surface, labeled on the capture antibody, or directly placed in the detection base solution. Then, based on the antigen antibody specific immune reaction, the ECL reagent forms an immune complex, which causes the change of ECL signal to realize the sensitive detection of target antigen. ECL direct immunization mode is to immobilize the antibody on the electrode surface directly. When the target antigen of different concentration appears, the antigen and antibody will have different degrees of immune reaction and then lead to different intensities of ECL response. Generally, with the increase of the concentration of target antigen, the formation of immune complex will hinder the electron transfer in the process of ECL, and the detected ECL signal will decrease, so as to realize the quantitative detection of target antigen. Electrochemiluminescence (ECL) DNA sensor is generally used to label ECL reagent directly or indirectly to form DNA probe at the end of double-stranded DNA. The probe can hybridize with target DNA strand through base complementary pairing. The change of ECL during hybridization can be detected to infer the concentration of target DNA.

\section{Experiment Materials and Methods}

3.1. Experimental Materials. $1 \mathrm{~g} \mathrm{HAuCl}_{4} \cdot 4 \mathrm{H}_{2} \mathrm{O}$ was dissolved in $412 \mathrm{~mL}$ three times distilled water to make a $0.2 \%(w / w)$ stock solution, stored at $4^{\circ} \mathrm{C}$ protected from light. Experimental materials include $\mathrm{N}$-(4-aminobutyl)$\mathrm{N}$-ethylisoluminol, 1-ethyl-(3-dimethylaminopropyl) car bodiimide hydrochloride, N-hydroxysuccinimide and 1,3-propanedithiol, immunoglobulin G, goat anti-Human $\mathrm{IgG}$, and bovine serum albumin. $5.6 \mathrm{mg} \mathrm{ABEI}$ was sissolve in $10 \mathrm{~mL}$ of $0.1 \mathrm{~mol} / \mathrm{L}$ phosphate buffer to prepare a $2 \mathrm{mmol} / \mathrm{LABEI}$ stock solution and stored at $4^{\circ} \mathrm{C}$ in the dark. The purity of all reagents is analytically pure, and the experimental water is Milli -Q ultrapure water.

\subsection{Preparation of Gold Electrode Modified by Nanogold.} After the glassware used in the experiment was soaked with aqua regia, it was washed with ultrapure water and air-dried. The bare gold electrode was first polished with fine sandpaper and suede, washed with ethanol and ultrapure water, and then dried with filter paper. The electrode was then electrochemically cleaned in sulfuric acid solution until the redox peak of the gold electrode was reproduced. The electrode was put into $0.1 \mathrm{~mol} / \mathrm{L} \mathrm{pH7.0} \mathrm{and} \mathrm{phosphate} \mathrm{buffer}$ solution was contained with a concentration of $1 \times 10^{-3} \mathrm{~mol} /$ $\mathrm{L}[\mathrm{Fe}(\mathrm{CN}) 6] 3-$ and $[\mathrm{Fe}(\mathrm{CN}) 6] 4-$ for cyclic voltammetry, Until the redox peak with a peak potential difference of less than $100 \mathrm{mV}$ appears, the electrode can be modified after washing with ultrapure water. The electrode is put into a $2 \mathrm{mmol} / \mathrm{L} \mathrm{1,3-propanedithiol} \mathrm{solution} \mathrm{at} \mathrm{room} \mathrm{temperature}$ for 20 hours and taken out. In order to remove the physically adsorbed 1,3-propanedithiol molecules, it should be repeatedly washed with ethanol and ultrapure water. On the electrode surface, a 1,3-propanedithiol self-assembled modified electrode was obtained. At $4^{\circ} \mathrm{C}$, the thiol-modified electrode was immersed in a $16 \mathrm{~nm}$ gold sol for 10 hours and then washed with ultrapure water to obtain a nanogoldmodified electrode for the next experiment. $100 \mathrm{~mL}$ of $0.3 \mathrm{mmol} / \mathrm{L} \mathrm{HAuCl} 4$ solution was heated to boiling. Under vigorous stirring, $5.0 \mathrm{~mL}$ of $1 \%(w / v)$ citrate solution was added quickly. After the mixture was heated to reflux for 30 minutes, the heating was stopped and stirring was continued for 15 minutes. After the synthetic sol was naturally cooled to room temperature, the cellulose particles with a pore size of $0.22 \mu \mathrm{m}$ were used to filter and remove the large particles in the sol and stored at $4^{\circ} \mathrm{C}$ in the dark.

\section{Development of Derivative Functionalized Gold Nanomaterial Industry and Its Application in Chemiluminescence Bioanalysis}

\subsection{Application of Electrochemiluminescence Immunosensor} in Real Human Serum Samples. The recovery results of IgG in human serum samples determined by ECL immunosensor are shown in Table 1.

The results showed that the recovery was between $89.6 \%$ and $117.8 \%$. Therefore, the sensor constructed in this work can be applied to the detection of IgG in human serum samples. The comparative analysis of ECL immunosensor and other immunoassay methods for the determination of $\operatorname{IgG}$ is shown in Figure 1.

Before detection, the sample should be diluted to the appropriate concentration, that is, the detection range of the 
TABLE 1: Recovery of IgG in human serum by electrochemiluminescence immunosensor.

\begin{tabular}{lccc}
\hline Background content $(\mathrm{ng} / \mathrm{ml})$ & Added concentration $(\mathrm{ng} / \mathrm{ml})$ & Detected concentration $(\mathrm{mean} \pm \mathrm{SD}, n=5)(\mathrm{ng} / \mathrm{ml})$ & Recovery ratio $(\%)$ \\
\hline 9.81 & 10 & $17.49 \pm 0.84$ & 89.9 \\
9.81 & 15 & $24.57 \pm 0.69$ & 115.7 \\
9.81 & 25 & $36.48 \pm 0.68$ & 119.4 \\
9.81 & 40 & $45.77 \pm 1.37$ & 113.6 \\
9.81 & 60 & $67.69 \pm 2.47$ & 116.4 \\
\hline
\end{tabular}

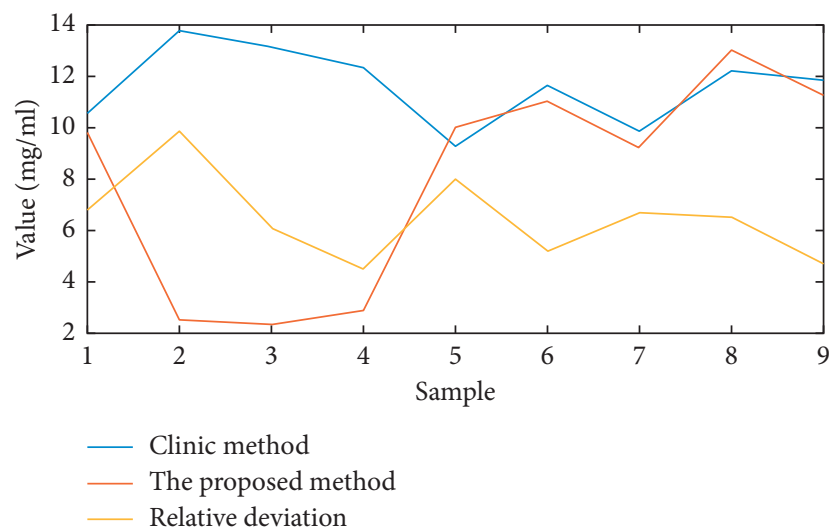

FIgURE 1: Comparison of electrochemiluminescence immunosensor with other immunoassay methods for the determination of IgG.

sensor. Compared with the existing clinical methods, the relative deviation of the two methods is less than $17 \%$. In the electrochemical way of two-step pulse, the immunosensor showed excellent electrochemiluminescence signal response in $0.01 \mathrm{~mol} / \mathrm{L}$ salt buffer containing $2 \mathrm{mmol} / \mathrm{L}$ hydrogen peroxide. The detection concentration range of human IgG was $10 \sim 150 \mathrm{ng} / \mathrm{m}$. The detection of IgG by the sensor was three orders of magnitude higher than other double antibody sandwich immunoassay methods based on the modified electrode as carrier. Although the sensitivity of this method is lower than that of homogeneous immunoassay, the presence of interferents in serum samples will have a great impact on luminescence in homogeneous immunoassay due to the influence of matrix. Therefore, it needs to be improved in practice.

4.2. ECL Assay of Thrombin by ECL Aptamer Sensor. The performance comparison between ECL aptamer sensor and other sensors is shown in Table 2.

Compared with other sensors, the proposed ECL sensor has lower detection limit and larger detection range, which indicates that the ECL sensor has higher sensitivity. The performance comparison and analysis between ECL aptamer sensor and other sensors is shown in Figure 2.

The reason for the low detection limit of the sensor is that AuNP has the effect similar to the electron channel, which can accelerate the electron transfer ability and increase the load of DNA and gqds; gqds has high ECL strength and good conductivity, which can realize ECL signal amplification; pica/fgns nanocomposites have the dual advantages of fgns and pica, providing larger specific surface area. It promoted the immobilization of bioactive molecules. The combination of the three can not only accelerate the electron conduction rate but also enhance the specific surface area of the electrode, which can effectively improve the detection effect of the sensor. The sensor has a wide linear range of $0.001-15 \mathrm{~nm}$ and a low detection limit of $0.06 \mathrm{pm}$. At the same time, the sensor shows good stability, selectivity, and reproducibility, which can be used to detect thrombin in real human serum samples. The sensor not only provides a simple method to detect thrombin but also expands the application range of pica/fgns nanocomposites in biological detection. This ECL strategy based on conductive polyindole derivatives also has potential application value in clinical diagnosis of other bioproteins.

4.3. Analysis of ECL Properties of Metal Ion Bifunctional Gold Nanoparticles. The $\mathrm{I}_{\mathrm{ECL}}$-E curve obtained by cyclic voltammetry of $\mathrm{Co}^{2+} /$ DTDTPA/ABEI-GNPs under air saturation condition is shown in Figure 3.

There is an obvious ECL peak in $\mathrm{Co}^{2+}$ /DTDTPA/ABEIGNPs at positive potential, while ABEI-GNPs hardly shows any luminescence. When $\mathrm{pH}$ is 12.4, $\mathrm{Co}^{2+} / \mathrm{DTDTPA} /$ ABEI-GNPs have a strong ECL peak at positive potential, while Abei GNPs have weak luminescence under this condition. The results show that the ECL intensity of $\mathrm{Co}^{2+} /$ DTDTPA/ABEI-GNPs is at least two orders of magnitude higher than ABEI-GNPs under neutral or alkaline conditions. Two ECL peaks, ECL- 1 and ECL-2 at $-0.89 \mathrm{v}$ and $0.74 \mathrm{v}$, were observed under cyclic voltammetry. These two peaks were related to the formation of OOH- and ABEI- by electrooxidation of dissolved oxygen as well as the formation of many intermediate products. The results showed that BF-GNPs had good ECL properties under neutral and 
TABle 2: Performance comparison between ECL aptamer and other sensors.

\begin{tabular}{lccc}
\hline Material science & Test method & Detection range $(\mathrm{nM})$ & Detection limit $(\mathrm{pM})$ \\
\hline $\mathrm{Ru}(\mathrm{bpy})_{3}^{2+}$ & ECL & $0.02-12$ & 6.8 \\
$\mathrm{CD} / \mathrm{AuNF}$ & $\mathrm{ECL}$ & $0.6-43$ & 82 \\
Fc-GNs & ECL & $0.7-27$ & 213 \\
Eu-MWCNTs & ECL & $0.003-5.2$ & 0.24 \\
Tris(bpyRu)- $\beta$-CD & ECL & $0.002-10.4$ & 0.14 \\
PTC-Tb & ECL & $0.006-10.7$ & 1.73 \\
GQDs/PICA/FGNs & ECL & $0.001-10.5$ & 0.06 \\
\hline
\end{tabular}

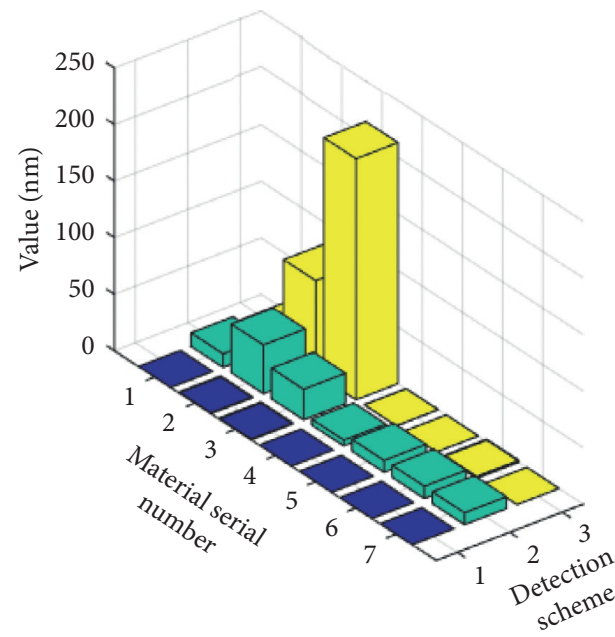

$\square$ Upper limit of detection range
Lower detection limit
Detection limit

Figure 2: Comparative analysis of the performance of the experimentally constructed ECL aptamer sensor and other sensors.

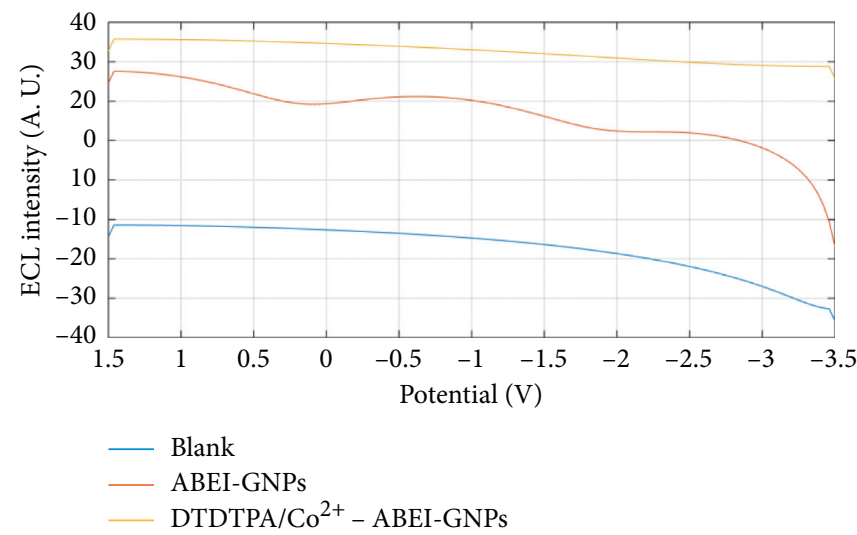

Figure 3: The $\mathrm{I}_{\mathrm{ECL}}-\mathrm{E}$ curves of $\mathrm{Co}^{2+} / \mathrm{DTDTPA} / \mathrm{ABEI}-\mathrm{GNPs}$ obtained by cyclic voltammetry under air saturation.

alkaline conditions, and the detection of bioactive substances under physiological conditions had important application potential.

4.4. Analysis of Chemiluminescence Mechanism. In the presence of catalyst, the chemiluminescence intensity of luminol reaction with hydrogen peroxide was significantly enhanced. The effect of different free radical scavengers on $\mathrm{CL}$ of luminol- $\mathrm{H}_{2} \mathrm{O}_{2}-\mathrm{MOF}-235 / \beta-\mathrm{CD}$ system is shown in Figure 4.

The chemiluminescence intensity was strongly inhibited by ascorbic acid and thiourea, indicating that a large amount of oh was produced in the reaction. At the same time, p-benzoquinone is usually used to detect $\mathrm{O}_{2}{ }^{\circ-}$. The results showed that p-benzoquinone inhibited the 


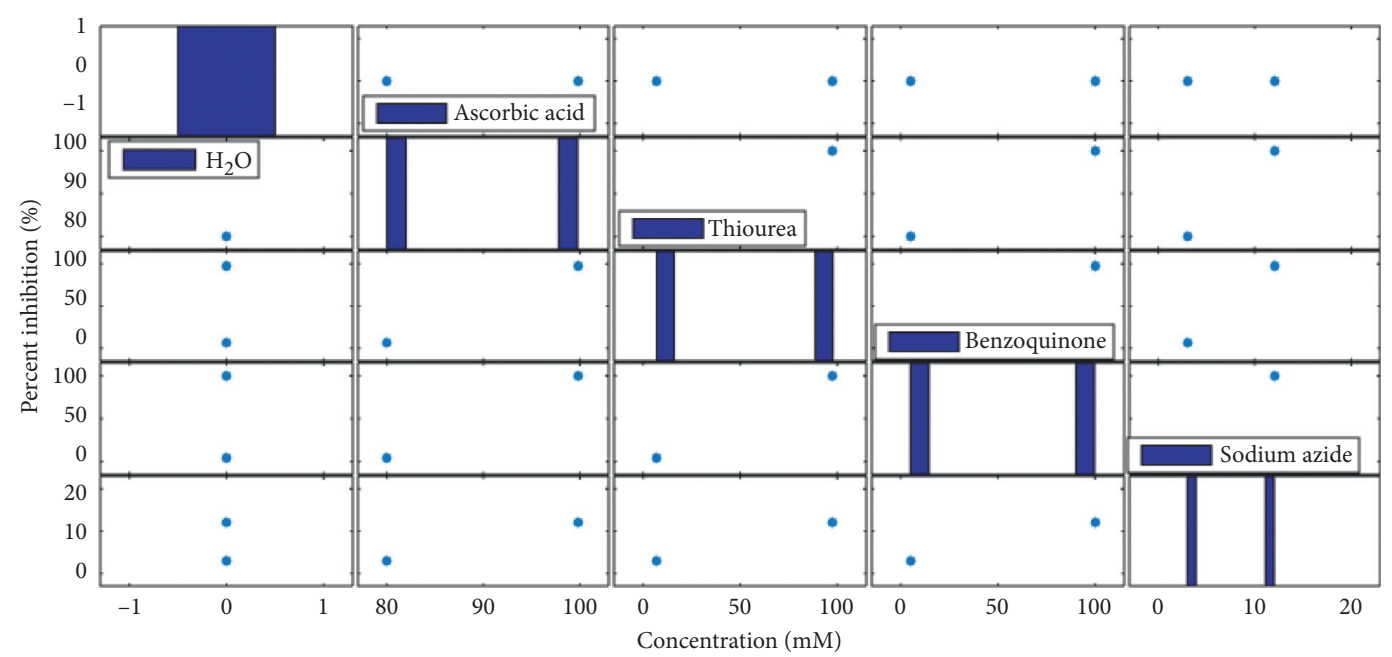

Figure 4: Effect of different free-radical scavengers on CL of the luminol- $\mathrm{h}_{2} \mathrm{O}_{2}-\mathrm{mof}-235 / \beta-\mathrm{CD}$ system.

chemiluminescence reaction of luminol-h2o2-mof-235/ $\beta$-CD system, and superoxide anion radical participated in the chemiluminescence reaction. In addition, sodium azide is an effective scavenger for singlet oxygen. The addition of $3 \mathrm{mM}$ sodium azide inhibited the chemiluminescence signal by $14 \%$, indicating that singlet oxygen played a secondary role in the process. Compared with mof-235 alone, mof-235/ $\beta$-CD exhibited higher catalytic activity due to the synergistic effect between $\beta-C D$ and mof-235. The gold nanomaterials synthesized by further research have excellent chemiluminescence activity and can be used to label biomolecules and prepare biological probes. The industrial development of derivative functionalized gold nanomaterials has broad application prospects in biological analysis.

\section{Conclusions}

This paper adopts the strategy of functionalizing MOF-235 with $\beta-\mathrm{CD}$, and the obtained MOF-235/ $\beta$-CD complex has been proved to be an effective catalyst in the hydrogen peroxide-Luminol CL system. In view of the advantages of functionalized MOF and chemiluminescence, it is believed that the success of this work will facilitate the future development of CL catalysts through MOF functionalization.

In this paper, the study of the effects of luminescent reagents found that luminol and isoluminol dual-functionalized nanophosphors have strong ECL emission without coreactive reagents. In addition, $\mathrm{Co}^{2+}$ /DTDTPA/ ABEI-GNPs have strong and stable ECL signals under double-order pulse conditions, indicating that the material has potential for analysis.

The electroluminescence functionalized gold nanoparticles with special morphology were synthesized by the seed-induced growth method using isoluminol as reducing agent in aqueous phase at room temperature. Compared with other gold nanomaterials, this gold nanomaterial not only has special morphology and special properties but also can be used in biochip connection, nanoelectronic devices, etc. and also has chemiluminescence activity and good biocompatibility. According to the morphology and properties of this material and its application in electrochemiluminescence biosensor platforms, gold nanomaterials, as a new type of material, can be used as a breakthrough in the development of new industries in Yunnan Province, China, to realize the differentiated development and application of nanomaterials. It provides new ideas for the construction of a modern industrial system in the central Yunnan urban agglomeration in Yunnan Province, China. This is the application of chemical methods to industrial development, which can provide targeted and efficient path suggestions for the development of emerging industries. It can be used in immune analysis and nucleic acid analysis and has broad application prospects. The application of chemical methods in emerging industries has direct and practical significance. As the kingdom of nonferrous metals, gold nanomaterials have a good development prospect in the central Yunnan urban agglomeration. They can become an important part of the local modern industrial system. The entry point of nanomaterials analysis requires innovation. This article finds that the application of gold nanomaterials in chemiluminescence bioanalysis has huge industrial development prospects, so the development possibilities of related industries are explored from the perspective of chemical analysis to realize management science and engineering disciplines and chemistry interdisciplinary integration.

\section{Data Availability}

The data used to support the findings of this study are included within the article. There is no other data to support this research.

\section{Conflicts of Interest}

The authors declare that there are no conflicts of interest regarding the study. 


\section{References}

[1] L. Wu, Y. Zhang, Y. Wang et al., "A paper-based electrochemiluminescence electrode as an aptamer-based cytosensor usingPtNi@carbon dots as nanolabels for detection of cancer cells and for in-situ screening of anticancer drugs," Microchimica Acta, vol. 183, no. 6, pp. 1873-1880, 2016.

[2] J. M. Sosenko, L. Yu, J. P. Krischer et al., "The use of electrochemiluminescence assays to predict autoantibody and glycemic progression toward type 1 diabetes in individuals with single autoantibodies," Diabetes Technology \& Therapeutics, vol. 19, no. 3, pp. 183-187, 2017.

[3] S. Carrara, F. Arcudi, M. Prato, and L. De Cola, "Amine-rich nitrogen-doped carbon nanodots as a platform for self-enhancing electrochemiluminescence," Angewandte Chemie International Edition, vol. 56, no. 17, pp. 4757-4761, 2017.

[4] H. Chen, H. Zhang, and R. Yuan, S. Chen, "Novel doublepotential electrochemiluminescence ratiometric strategy in enzyme-based inhibition biosensing for sensitive detection of organophosphorus pesticides," Analytical Chemistry, vol. 89, no. 5, pp. 2823-2829, 2017.

[5] Q. Liu, Y.-J. Peng, J.-C. Xu et al., "Label-free electrochemiluminescence aptasensor for highly sensitive detection of acetylcholinesterase based on Au-Nanoparticle-Functionalized g- $\mathrm{C}_{3} \mathrm{~N}_{4}$ nanohybrid," Chemelectrochem, vol. 4, no. 7, pp. 1768-1774, 2017.

[6] Z. Li, Y. Zhou, L. Peng, D. Yan, and M. Wei, "A switchable electrochromism and electrochemiluminescence bifunctional sensor based on the electro-triggered isomerization of spiropyran/layered double hydroxides," Chemical Communications, vol. 53, no. 63, pp. 8862-8865, 2017.

[7] P. Zhang, Z. Li, H. Wang, Y. Zhuo, R. Yuan, and Y. Chai, "DNA nanomachine-based regenerated sensing platform: a novel electrochemiluminescence resonance energy transfer strategy for ultra-high sensitive detection of microRNA from cancer cells," Nanoscale, vol. 9, no. 6, pp. 2310-2316, 2017.

[8] S. Li, C. Liu, B. Han, and J. Luo, G. Yin, "An electrochemiluminescence aptasensor switch for aldicarb recognition via ruthenium complex-modified dendrimers on multiwalled carbon nanotubes," Microchimica Acta, vol. 184, no. 6, pp. 1669-1675, 2017.

[9] J. Zhang, S. Devaramani, D. Shan, and X. Lu, "Electrochemiluminescence behavior of meso-tetra(4-sulfonatophenyl)porphyrin in aqueous medium: its application for highly selective sensing of nanomolar $\mathrm{Cu} 2+$," Analytical and Bioanalytical Chemistry, vol. 408, no. 25, pp. 7155-7163, 2016.

[10] K. Shao, B. Wang, S. Ye et al., "Signal-amplified near-infrared ratiometric electrochemiluminescence aptasensor based on multiple quenching and enhancement effect of graphene/gold nanorods/G-quadruplex," Analytical Chemistry, vol. 88, no. 16, pp. 8179-8187, 2016.

[11] G. Jie, K. Chen, X. Wang, and Z. Lu, "Dual-stabilizer-capped CdSe quantum dots for "Off-On" electrochemiluminescence biosensing of thrombin by target-triggered multiple amplification," RSC Advances, vol. 6, no. 3, pp. 2065-2071, 2016.

[12] M. L. D. O. Pereira, D. Grasseschi, and H. E. Toma, "Photocatalytic activity of reduced graphene oxide-gold nanoparticle nanomaterials: interaction with asphaltene and conversion of a model compound," Energy \& Fuels, vol. 32, no. 3, pp. 2673-2680, 2018.

[13] Q. H. Quach and J. C. Y. Kah, "Non-specific adsorption of complement proteins affects complement activation pathways of gold nanomaterials," Nanotoxicology, vol. 11, no. 3, pp. 382-394, 2017.
[14] M. Alrahili, R. Peroor, V. Savchuk, K. McNear, and A. Pinchuk, "Morphology dependence in photothermal heating of gold nanomaterials with near-infrared laser," The Journal of Physical Chemistry C, vol. 124, no. 8, pp. 4755-4763, 2020.

[15] A. K. Alanazi, A. Mezni, N. B. Saber et al., "Aquatic ecotoxicity effects of gold/titania based nanomaterials," Journal of Biomaterials and Tissue Engineering, vol. 9, no. 2, pp. 262-267, 2019.

[16] B. Wongyun, J. Soeun, N. M. Tran, and H. Yo, "Synthesis and application of dendritic fibrous nanosilica/gold hybrid nanomaterials," ChemistryOpen, vol. 7, no. 5, pp. 349-355, 2018.

[17] S. Chakraborty, R. C. Rocha, A. Desireddy et al., "Gold nanocluster formation using morpholino oligomer as template and assembly agent within hybrid bio-nanomaterials," RSC Advances, vol. 6, no. 93, pp. 90624-90630, 2016.

[18] N. M. Ushakov, M. Y. Vasil'kov, and V. R. Shaturnuy, "Influence of the pore structure on the electron barrier height of metal-ceramic nanomaterials based on gold-anodic aluminum oxide," Semiconductors, vol. 52, no. 16, pp. 2078-2080, 2018.

[19] Y. Shi and B. Zhang, "Correction: recent advances in transition metal phosphide nanomaterials: synthesis and applications in hydrogen evolution reaction," Chemical Society Reviews, vol. 45, no. 6, p. 1781, 2016.

[20] J. Fernández-Lodeiro, B. Rodríguez-González, H. M. Santos et al., "Unraveling the organotellurium chemistry applied to the synthesis of gold nanomaterials," Acs Omega, vol. 1, no. 6, pp. 1314-1325, 2016.

[21] T. Y. Sun, N.A. Bornhöf, K. Hungerbühler, and B. Nowack, "Dynamic probabilistic modeling of environmental emissions of engineered nanomaterials," Environmental Science \& Technology, vol. 50, no. 9, pp. 4701-4711, 2016.

[22] S. Choi, H. Lee, R. Ghaffari, T. Hyeon, and D.-H. Kim, "Recent advances in flexible and stretchable bio-electronic devices integrated with nanomaterials," Advanced Materials, vol. 28, no. 22, pp. 4203-4218, 2016.

[23] G. C. Zhang, J. Zhong, M. Xu et al., "Ternary $\mathrm{BiVO}_{4} / \mathrm{NiS} / \mathrm{Au}$ nanocomposites with efficient charge separations for enhanced visible light photocatalytic performance," Chemical Engineering Journal, vol. 375, Article ID 122093, 2019.

[24] M. Feng, J. Gu, G. C. Zhang et al., "Homogeneous nickel bicarbonate nanocrystals as electrode materials for highperformance asymmetric supercapacitors," Journal of Solid State Chemistry, vol. 282, Article ID 121084, 2020.

[25] L. Simin, L. Meng, L. Debao, Y. Yang, P. Yang et al., "Selfassembly of Au nanoparticles and quantum dots by functional sol-gel silica layers," Journal of Nanoscience and Nanotechnolog, vol. 18, no. 1, pp. 288-295, 2018.

[26] C. Jin, M. Feng, J. Zhong et al., "Insights into mechanism of size-controlled synthesis of $\mathrm{CH}_{3} \mathrm{NH}_{3} \mathrm{PbBr}_{3}$ perovskite quantum dots and large nanoparticles with tunable optical properties," Organic Electronics, vol. 82, Article ID 105712, 2020. 